\title{
Multiplicity of solutions for a class of quasi- linear elliptic equation involving the critical Sobolev and Hardy exponents
}

Sihua Liang and Jihui Zhang

\begin{abstract}
In this paper, by using concentration-compactness principle and a new version of the symmetric mountain-pass lemma due to Kajikiya (J Funct Anal 225:352-370, 2005), infinitely many small solutions are obtained for a class of quasilinear elliptic equation with singular potential
\end{abstract}

$$
-\Delta_{p} u-\mu \frac{|u|^{p-2} u}{|x|^{p}}=\frac{|u|^{p^{*}(s)-2} u}{|x|^{s}}+\lambda f(x, u), \quad u \in H_{0}^{1, p}(\Omega) .
$$

Mathematics Subject Classification (2000). 35J60, 35J92, 35J25.

Keywords. p-Laplacian, Hardy-Sobolev critical exponent, Variational method, Compactness.

\section{Introduction}

In this paper, we study the existence of infinitely many small solutions for the following quasilinear elliptic equation with singular potential

$$
\left\{\begin{array}{l}
-\Delta_{p} u-\mu \frac{|u|^{p-2} u}{|x|^{p}}=\frac{|u|^{p^{*}(s)-2} u}{|x|^{s}}+\lambda f(x, u), \quad x \in \Omega, \\
u=0, \quad x \in \partial \Omega
\end{array}\right.
$$

where $1<p<N,-\Delta_{p} u:=-\operatorname{div}\left(|\nabla u|^{p-2} \nabla u\right)$ is the $p$-Lapace, $0 \leq \mu<\bar{\mu}:=$ $\left(\frac{N-p}{p}\right)^{p}, \lambda$ is a positive parameter, and $\Omega \subset R^{N}(N \geq 3)$ is an open bounded domain with smooth boundary, $0 \in \Omega, 0 \leq s \leq p<N, p^{*}(s)=\frac{(N-s) p}{N-p}$ is the so called Hardy-Sobolev critical exponent. When $s=0, p^{*}(0)=p^{*}=\frac{N p}{N-p}$ is the Sobolev critical exponent and if $s=p, p^{*}(p)=p$ is the Hardy critical exponent. 
When $p=2$. Elliptic equations with critical exponent have been considered by many authors since the pioneer work by Brezis and Nirenberg [1] in case $s=0$ and $\mu=0$. When a singular potential is concerned, He and Zou [2] proved that the existence infinitely many small solutions which converge to zero. In [3], by using variational arguments and moving plane method, the author proved some results about existence, uniqueness and qualitative behavior of positive solutions to a class of equations with singular coefficient and critical exponent (see [4-8]).

When $p \neq 2$. Ghoussoub and Yuan [9] obtained the existence of infinitely many nontrivial solutions for Hardy-Sobolev subcritical case and Hardy critical case by establishing Palais-Smale type conditions around appropriate chosen dual sets in bounded domain. Besides, although there are a lot of papers about the singular problems with Hardy-Sobolev critical exponents (the case that $p \neq 2$ ) (see $[10,11]$ ). But there are few results dealing with the case the general form $f(x, t)$. In [12], the authors considered the Eq. (1.1) in case $\lambda f(x, u)=u^{p^{*}-1}$, in which also the case $\mu<0$ is considered, as well as existence of minimizers. In [13], Roberta Musina studied existence and multiplicity results for a weighted $p$-Laplace equation involving Hardy potentials and critical nonlinearities, in which there is a survey on the most recent results, as well as new existence and multiplicity results. When $f(x, u)=\frac{|u|^{q-2}}{|x|^{s}} u$, the existence of positive solutions for the Eq. (1.1) are obtained in [14]. Chen and Li [15] obtained that the existence of infinitely many solutions by using minimax procedure in the case $\mu=0$ and $f(x, u)=k(x)|u|^{r-2} u\left(1<r<\frac{N p}{N-p}\right)$. But they did not give any further information on the sequence of solutions.

Recently, Kajikiya [16] established a critical point theorem related to the symmetric mountain pass lemma and applied to a sublinear elliptic equation. But there are no such results on singular quasilinear elliptic problems with critical Sobolev exponents and Hardy terms (1.1).

Motivated by reasons above, the aim of this paper is to show that the existence of infinitely many solutions of problem (1.1), and there exists a sequence of infinitely many arbitrarily small solutions converging to zero by using a new version of the symmetric mountain-pass lemma due to Kajikiya [16]. In order to use the symmetric mountain-pass lemma, there are many difficulties. The main one in solving the problem is a lack of compactness which can be illustrated by the fact that the embedding of $H_{0}^{1, p}(\Omega)$ into $L^{p^{*}}(\Omega)$ is no longer compact. Hence the concentration-compactness principle is used here to overcome the difficulty.

$u \in H_{0}^{1, p}(\Omega)$ is said to be a solutions of problem (1.1) if $u$ satisfies

$$
\int_{\Omega}\left(|\nabla u|^{p-2} \nabla u \cdot \nabla v-\mu \frac{|u|^{p-2} u v}{|x|^{p}}-\frac{|u|^{p^{*}(s)-2} u v}{|x|^{s}}-\lambda f(x, u) v\right) d x=0
$$

for all $v \in H_{0}^{1, p}(\Omega)$.

In the case $\mu=0$, problem (1.1) is related to the well known SobolevHardy inequalities, which is essentially due to Caffarelli, Kohn and Nirenberg (see [17]), 


$$
\left(\int_{R^{N}} \frac{|u|^{q}}{|x|^{s}}\right)^{\frac{p}{q}} \leq C_{q, s, p} \int_{R^{N}}|\nabla u|^{p} d x, \quad \forall u \in H_{0}^{1, p}(\Omega),
$$

where $p \leq q \leq p^{*}$. For sharp constants and extremal functions, see [9]. As $q=s=p$, the above Sobolev inequality becomes the well known Hardy inequality (see $[9,17,18]$ ),

$$
\int_{\Omega} \frac{|u|^{p}}{|x|^{p}} \leq \frac{1}{\bar{\mu}} \int_{\Omega}|\nabla u|^{p} d x, \quad \forall u \in H_{0}^{1, p}(\Omega) .
$$

In this paper, we use the norm

$$
\|u\|=\|u\|_{H_{0}^{1, p}(\Omega)}:=\left(\int_{\Omega}\left(|\nabla u|^{p}-\mu \frac{|u|^{p}}{|x|^{p}}\right) d x\right)^{\frac{1}{p}},
$$

by (1.2), this norm is equivalent to the usual norm $\left(\int_{\Omega}|\nabla u|^{p} d x\right)^{1 / p}$. As a consequence of Hardy inequality (1.2), the elliptic operator $L:=\left(-|\nabla \cdot|^{p-2} \nabla \cdot\right)-$ $\frac{\mu}{|x|^{p}}|\cdot|^{p-2}$. is positive in $H_{0}^{1, p}(\Omega)$ if $0 \leq \mu<\bar{\mu}$.

By the Hardy inequality and the Sobolev-Hardy inequality, for $0 \leq \mu$ $<\bar{\mu}, 0 \leq s<p$ and $p \leq r \leq p^{*}(s)$, we can define the Sobolev-Hardy constant:

$$
A_{\mu, s, r}(\Omega):=\inf _{u \in H_{0}^{1, p}(\Omega) \backslash\{0\}} \frac{\int_{\Omega}\left(|\nabla u|^{p}-\mu \frac{|u|^{p}}{|x|^{p}}\right) d x}{\left(\int_{\Omega} \frac{|u|^{r}}{|x|^{s}} d x\right)^{\frac{p}{r}}} .
$$

In the important case where $r=p^{*}(s)$, we shall simply denote $A_{\mu, s, p^{*}(s)}$ as $A_{s}$. Note $A_{\mu, 0}$ is the best constant in the Sobolev inequality, i.e.,

$$
A_{\mu, 0}(\Omega):=\inf _{u \in H_{0}^{1, p}(\Omega) \backslash\{0\}} \frac{\int_{\Omega}\left(|\nabla u|^{p}-\mu \frac{|u|^{p}}{|x|^{p}}\right) d x}{\left(\int_{\Omega}|u|^{p^{*}} d x\right)^{\frac{p}{p^{*}}}} .
$$

Note that $A_{\mu, 0, p}(\Omega)$ is nothing but the first eigenvalue of the positive operator $L$ in $H_{0}^{1, p}(\Omega)$ :

$$
\lambda_{1}(\Omega):=\inf _{u \in H_{0}^{1, p}(\Omega) \backslash\{0\}} \frac{\int_{\Omega}\left(|\nabla u|^{p}-\mu \frac{|u|^{p}}{|x|^{p}}\right) d x}{\left(\int_{\Omega}|u|^{p} d x\right)} .
$$

The energy functional corresponding to problem (1.1) is defined as follows,

$$
I(u)=\frac{1}{p} \int_{\Omega}\left(|\nabla u|^{p}-\mu \frac{|u|^{p}}{|x|^{p}}\right) d x-\frac{1}{p^{*}(s)} \int_{\Omega} \frac{|u|^{p^{*}(s)}}{|x|^{s}} d x-\lambda \int_{\Omega} F(x, u) d x,
$$

then $I(u)$ is well defined on $H_{0}^{1, p}(\Omega)$. Standard arguments [19] show that $I(u)$ belongs to $C^{1}\left(H_{0}^{1, p}(\Omega), R\right)$. The solutions of problem (1.1) are then the critical points of the functional $I$.

The main result of this paper is as follows.

Theorem 1.1. Suppose that $f(x, u)$ satisfies the following conditions:

$\left(\mathrm{H}_{1}\right) f(x, u) \in C(\Omega \times R, R), f(x,-u)=-f(x, u)$ for all $u \in R$;

$\left(\mathrm{H}_{2}\right) \lim _{|u| \rightarrow \infty} \frac{f(x, u)}{|u|^{p^{*}(s)-1}}=0$ uniformly for $x \in \Omega$;

$\left(\mathrm{H}_{3}\right) \lim _{|u| \rightarrow 0^{+}} \frac{f(x, u)}{u^{p-1}}=\infty$ uniformly for $x \in \Omega$. 
There then exists $\lambda^{*}>0$ such that for any $\lambda \in\left(0, \lambda^{*}\right)$, problem (1.1) has a sequence of non-trivial solutions $\left\{u_{n}\right\}$ and $u_{n} \rightarrow 0$ as $n \rightarrow \infty$.

Remark 1.1. When $p=2, \mu=0$ and $s=0$, the authors in [20] proved the existence of infinitely many solutions for $(1.1)$ under conditions $\left(H_{1}\right)-\left(H_{3}\right)$ and

$\left(\mathrm{H}_{4}\right) \frac{1}{2} f(x, u) u-F(x, u) \geq a-b|u|^{2^{*}}$ for almost every $x \in \Omega$ and $u \in R$ where $F(x, u)=\int_{0}^{u} f(x, t) d t, \quad b \geq 0, a \leq 0$.

But they did not give any further information on the sequence of solutions. When $p=2$, the authors in [2] proved the existence of infinitely many small solutions for $(1.1)$ under conditions $\left(H_{1}\right)-\left(H_{3}\right)$. In this paper, we shall prove that this sequence of solutions may converge to zero for (1.1).

Remark 1.2. In this paper, the nonlinearity $f(x, u)$ need not satisfy condition $\left(H_{4}\right)$ as in [20]. Furthermore, we consider more general nonlinearity than is considered in $[14,15]$. Hence, we make a improvement of the main results of $[2,14,15,20]$.

Definition 1.1. A $C^{1}$ functional $I$ on Banach space $X$ satisfies the Palais-Smale condition at level $c\left((P S)_{c}\right.$, for short) if every sequence $\left\{u_{n}\right\}$ satisfying

$$
I\left(u_{n}\right) \rightarrow c \text { and } I^{\prime}\left(u_{n}\right) \rightarrow 0,
$$

contains a convergent subsequence.

\section{Preliminary lemmas}

Denote $\mathcal{M}^{+}$as a cone of positive finite Radon measure. Since the proof of the following result is similar to Lions $[21,22]$ and is an adaptation of lemma by Smets [24], we just sketch the proof here.

Lemma 2.1. Let $0 \leq s \leq p<N$ and $\left\{u_{n}\right\} \subset H_{0}^{1, p}(\Omega)$ be a bounded sequence, going if necessary to subsequence, we may assume that $u_{n} \rightarrow u$ in $H_{0}^{1, p}(\Omega)$, $\left|\nabla u_{n}\right|^{p} \rightarrow \zeta$ in $\mathcal{M}^{+}, \frac{\left|u_{n}\right|^{p^{*}(s)}}{|x|^{s}} \rightarrow \nu$ in $\mathcal{M}^{+}$. Define

$$
\begin{aligned}
\zeta_{\infty} & :=\lim _{R \rightarrow \infty} \varlimsup_{n \rightarrow \infty} \int_{\Omega \cap\{x \in \Omega:|x|>R\}}\left|\nabla u_{n}\right|^{p} d x, \\
\nu_{\infty} & :=\lim _{R \rightarrow \infty} \varlimsup_{n \rightarrow \infty} \int_{\Omega \cap\{x \in \Omega:|x|>R\}} \frac{\left|u_{n}\right|^{p^{*}(s)}}{|x|^{s}} d x .
\end{aligned}
$$

Then there exist a, at most, countable index set $J$ and a collection of points $\left\{x_{j}\right\}, j \in J$, in $\Omega$ such that

(i) $\zeta_{\infty} \geq A_{s} \nu_{\infty}^{p / p^{*}(s)}$;

(ii) $\nu=\frac{|u|^{p^{*}(s)}}{|x|^{s}}+\sum \delta_{x_{j}} \nu_{j}, \nu_{j}>0, \zeta=|\nabla u|^{p}+\sum \delta_{x_{j}} A_{s} \nu_{j}^{p / p^{*}(s)}$;

(iii) $\zeta_{j} \geq \delta_{x_{j}} A_{s} \nu_{j}^{p / p^{*}(s)}$;

(iv) $\lim _{n \rightarrow \infty} \int_{\Omega \cap\{x \in \Omega:|x|>R\}} \frac{\left|u_{n}\right|^{p^{*}(s)}}{|x|^{s}} d x=\int_{\Omega \cap\{x \in \Omega:|x|>R\}} \frac{|u|^{p^{*}(s)}}{|x|^{s}} d x+\|\nu\|+\nu_{\infty}$. 
Under assumption $\left(\mathrm{H}_{2}\right)$, we have

$$
f(x, u) u=o\left(\frac{|u|^{p^{*}(s)}}{|x|^{s}}\right), \quad F(x, u)=o\left(\frac{|u|^{p^{*}(s)}}{|x|^{s}}\right),
$$

which means that, for all $\varepsilon>0$, there exist two constants $a(\varepsilon), b(\varepsilon)>0$ which depend on $\varepsilon$ such that

$$
\begin{aligned}
& |f(x, u) u| \leq a(\varepsilon)+\varepsilon \frac{|u|^{p^{*}(s)}}{|x|^{s}}, \\
& |F(x, u)| \leq b(\varepsilon)+\varepsilon \frac{|u|^{p^{*}(s)}}{|x|^{s}} .
\end{aligned}
$$

Hence,

$$
F(x, u)-\frac{1}{p} f(x, u) u \leq c(\varepsilon)+\varepsilon \frac{|u|^{p^{*}(s)}}{|x|^{s}},
$$

for some constant $c(\varepsilon)>0$ which depend on $\varepsilon$.

Lemma 2.2. Assume condition $\left(\mathrm{H}_{2}\right)$ holds. Then for any $\lambda>0$, the functional $I$ satisfies the local $(P S)_{c}$ condition in

$$
c \in\left(-\infty, \frac{p-s}{p(N-s)} A_{s}^{\frac{N-s}{p-s}}-\lambda c\left(\frac{p-s}{2 p \lambda(N-s)}\right)|\Omega|\right)
$$

in the following sense: if

$$
I\left(u_{n}\right) \rightarrow c<\frac{p-s}{p(N-s)} A_{s}^{\frac{N-s}{p-s}}-\lambda c\left(\frac{p-s}{2 p \lambda(N-s)}\right)|\Omega|
$$

and $I^{\prime}\left(u_{n}\right) \rightarrow 0$ for some sequence in $H_{0}^{1, p}(\Omega)$, then $\left\{u_{n}\right\}$ contains a subsequence converging strongly in $H_{0}^{1, p}(\Omega)$.

Proof. Let $\left\{u_{n}\right\}$ be a sequence in $H_{0}^{1, p}(\Omega)$ such that

$$
\begin{aligned}
I\left(u_{n}\right)= & \frac{1}{p} \int_{\Omega}\left(\left|\nabla u_{n}\right|^{p}-\mu \frac{\left|u_{n}\right|^{p}}{|x|^{p}}\right) d x-\frac{1}{p^{*}(s)} \int_{\Omega} \frac{\left|u_{n}\right|^{p^{*}(s)}}{|x|^{s}} d x \\
& -\lambda \int_{\Omega} F\left(x, u_{n}\right) d x \\
= & c+o(1)
\end{aligned}
$$

and

$$
\begin{aligned}
\left\langle I^{\prime}\left(u_{n}\right), u_{n}\right\rangle= & \int_{\Omega}\left(\left|\nabla u_{n}\right|^{p}-\mu \frac{\left|u_{n}\right|^{p}}{|x|^{p}}\right) d x-\int_{\Omega} \frac{\left|u_{n}\right|^{p^{*}(s)}}{|x|^{s}} d x \\
& -\lambda \int_{\Omega} f\left(x, u_{n}\right) u_{n} d x \\
= & o(1)\left\|u_{n}\right\| .
\end{aligned}
$$


It follows from (2.4) and (2.5) that

$$
\begin{aligned}
& I\left(u_{n}\right)-\frac{1}{p}\left\langle I^{\prime}\left(u_{n}\right), u_{n}\right\rangle \\
& \quad=\left(\frac{1}{p}-\frac{1}{p^{*}(s)}\right) \int_{\Omega} \frac{\left|u_{n}\right|^{p^{*}(s)}}{|x|^{s}} d x-\lambda \int_{\Omega}\left[F\left(x, u_{n}\right)-\frac{1}{p} f\left(x, u_{n}\right) u_{n}\right] d x \\
& \quad=c+o(1)\left\|u_{n}\right\|,
\end{aligned}
$$

i.e.,

$\frac{p-s}{p(N-s)} \int_{\Omega} \frac{\left|u_{n}\right|^{p^{*}(s)}}{|x|^{s}} d x=\lambda \int_{\Omega}\left[F\left(x, u_{n}\right)-\frac{1}{p} f\left(x, u_{n}\right) u_{n}\right] d x+c+o(1)\left\|u_{n}\right\|$.

Then by (2.3), we obtain

$$
\left(\frac{p-s}{p(N-s)}-\lambda \varepsilon\right) \int_{\Omega} \frac{\left|u_{n}\right|^{p^{*}(s)}}{|x|^{s}} d x \leq \lambda c(\varepsilon)|\Omega|+c+o(1)\left\|u_{n}\right\| .
$$

Setting $\varepsilon=(p-s) / 2 p(N-s) \lambda$, we get

$$
\int_{\Omega} \frac{\left|u_{n}\right|^{p^{*}(s)}}{|x|^{s}} d x \leq M+o(1)\left\|u_{n}\right\|
$$

where $o(1) \rightarrow 0$ and $M$ is a some positive number. On the other hand, by (2.2) and (2.6), we have

$$
\begin{aligned}
c+o(1)\left\|u_{n}\right\|= & I\left(u_{n}\right) \\
= & \frac{1}{p} \int_{\Omega}\left(\left|\nabla u_{n}\right|^{p}-\mu \frac{\left|u_{n}\right|^{p}}{|x|^{p}}\right) d x-\frac{1}{p^{*}(s)} \int_{\Omega} \frac{\left|u_{n}\right|^{p^{*}(s)}}{|x|^{s}} d x \\
& -\lambda \int_{\Omega} F\left(x, u_{n}\right) d x \\
\geq & \frac{1}{p}\left\|u_{n}\right\|^{p}-\lambda b(\varepsilon)|\Omega|-\left[\frac{1}{p^{*}(s)}+\lambda \varepsilon\right] \int_{\Omega} \frac{\left|u_{n}\right|^{p^{*}(s)}}{|x|^{s}} d x .
\end{aligned}
$$

Thus (2.6) and (2.7) imply that $\left\{u_{n}\right\}$ is bounded in $H_{0}^{1, p}(\Omega)$. Therefore we can assume that $u_{n} \rightarrow u$ in $H_{0}^{1, p}(\Omega),\left|\nabla u_{n}\right|^{p} \rightarrow \zeta$ in $\mathcal{M}^{+}, \frac{\left|u_{n}\right|^{p^{*}(s)}}{|x|^{s}} \rightarrow \nu$ in $\mathcal{M}^{+}$. Let $x_{j}$ be a singular point of the measures $\zeta$ and $\nu$, define a function $\phi(x) \in C_{0}^{\infty}(\Omega)$ such that $\phi(x)=1$ in $B\left(x_{j}, \varepsilon\right), \phi(x)=0$ in $\Omega \backslash B\left(x_{j}, 2 \varepsilon\right)$ and $|\nabla \phi| \leq 2 / \varepsilon$ in $\Omega$. Then $\left\{\phi u_{n}\right\}$ is bounded in $H_{0}^{1, p}(\Omega)$. Obviously, $\left\langle I^{\prime}\left(u_{n}\right), u_{n} \phi\right\rangle \rightarrow 0$, i.e.,

$$
\begin{aligned}
& \lim _{n \rightarrow \infty}\left[\int_{\Omega}\left(\left|\nabla u_{n}\right|^{p}-\mu \frac{\left|u_{n}\right|^{p}}{|x|^{p}}\right) \phi d x-\int_{\Omega} \frac{\left|u_{n}\right|^{p^{*}(s)}}{|x|^{s}} \phi d x-\lambda \int_{\Omega} f\left(x, u_{n}\right) u_{n} \phi d x\right] \\
& =-\lim _{n \rightarrow \infty} \int_{\Omega}\left(u_{n}\left|\nabla u_{n}\right|^{p-2} \nabla u_{n} \nabla \phi\right) d x
\end{aligned}
$$


On the other hand, by Hölder inequality and boundedness of $\left\{u_{n}\right\}$, it implies that

$$
\begin{aligned}
0 & \leq\left.\lim _{\varepsilon \rightarrow 0} \lim _{n \rightarrow \infty}\left|\int_{\Omega} u_{n}\right| \nabla u_{n}\right|^{p-2} \nabla u_{n} \nabla \phi d x \mid \\
& \leq \lim _{\varepsilon \rightarrow 0} \lim _{n \rightarrow \infty}\left(\int_{\Omega}\left|u_{n}\right|^{p}|\nabla \phi|^{p} d x\right)^{\frac{1}{p}}\left(\int_{\Omega}\left|\nabla u_{n}\right|^{p} d x\right)^{\frac{p-1}{p}} \\
& \leq C \lim _{\varepsilon \rightarrow 0}\left(\int_{\Omega}|u|^{p}|\nabla \phi|^{p} d x\right)^{\frac{1}{p}} \\
& \leq C \lim _{\varepsilon \rightarrow 0}\left(\int_{B\left(x_{j}, \varepsilon\right)}|\nabla \phi|^{N} d x\right)^{\frac{1}{N}}\left(\int_{B\left(x_{j}, \varepsilon\right)}|u|^{p^{*}} d x\right)^{\frac{1}{p^{*}}} \\
& \leq C \lim _{\varepsilon \rightarrow 0}\left(\int_{B\left(x_{j}, \varepsilon\right)}|u|^{p^{*}} d x\right)^{\frac{1}{p^{*}}}=0
\end{aligned}
$$

and

$$
0 \leq \lim _{\varepsilon \rightarrow 0} \lim _{n \rightarrow \infty}\left|\int_{\Omega} \frac{\left|u_{n}\right|^{p}}{|x|^{p}} \phi d x\right| \leq \lim _{\varepsilon \rightarrow 0} \lim _{n \rightarrow \infty}\left|\int_{B\left(x_{j}, \varepsilon\right)} \frac{\left|u_{n}\right|^{p}}{|| x_{j}|-\varepsilon|^{p}} \phi d x\right|=0 .
$$

From (2.8) to (2.10), we get

$$
0=\lim _{\varepsilon \rightarrow 0}\left[\int_{\Omega} \phi d \zeta-\int_{\Omega} \phi d \nu-\lambda \int_{\Omega} f\left(x, u_{n}\right) u_{n} \phi d x\right]=\zeta_{j}-\nu_{j} .
$$

Combing this with Lemma 2.1(iii), we obtain that $\nu_{j} \geq A_{s} \nu_{j}^{\frac{p}{p^{*}(s)}}$. This result implies that

$$
\nu_{j}=0 \quad \text { or } \quad \nu_{j} \geq A_{s}^{\frac{N-s}{p-s}} .
$$

If the second case $\nu_{j} \geq A_{s}^{\frac{N-s}{p-s}}$ holds, for some $j \in J$, then by using Lemma 2.1 and the Hölder inequality, we have

$$
\begin{aligned}
c & =\lim _{n \rightarrow \infty}\left(I\left(u_{n}\right)-\frac{1}{p}\left\langle I^{\prime}\left(u_{n}\right), u_{n}\right\rangle\right) \\
& =\left(\frac{1}{p}-\frac{1}{p^{*}(s)}\right) \int_{\Omega} \frac{\left|u_{n}\right|^{p^{*}(s)}}{|x|^{s}} d x-\lambda \int_{\Omega}\left[F\left(x, u_{n}\right)-\frac{1}{p} f\left(x, u_{n}\right) u_{n}\right] d x \\
& =\frac{p-s}{p(N-s)} \int_{\Omega} d \nu-\lambda \int_{\Omega}\left[F(x, u)-\frac{1}{p} f(x, u) u\right] d x \\
& \geq\left(\frac{p-s}{p(N-s)}-\lambda \varepsilon\right) \int_{\Omega} \frac{|u|^{p^{*}(s)}}{|x|^{s}} d x+\frac{p-s}{p(N-s)} A_{s}^{\frac{N-s}{p-s}}-\lambda c(\varepsilon)|\Omega| \\
& \geq \frac{p-s}{p(N-s)} A_{\mu}^{\frac{N-s}{p-s}}-\lambda c\left(\frac{p-s}{2 p \lambda(N-s)}\right)|\Omega|,
\end{aligned}
$$


where $\varepsilon=(p-s) / 2 p \lambda(N-s)$. This is impossible. Consequently, $\nu_{j}=0$ for all $j \in J$ and hence

$$
\int_{\Omega} \frac{\left|u_{n}\right|^{p^{*}(s)}}{|x|^{s}} d x \rightarrow \int_{\Omega} \frac{|u|^{p^{*}(s)}}{|x|^{s}} d x .
$$

Now $u_{n} \rightarrow u$ in $H_{0}^{1, p}(\Omega)$ and Brezis-Lieb Lemma [23] implies that

$$
\lim _{n \rightarrow \infty} \int_{\Omega} \frac{\left|u_{n}-u\right|^{p^{*}(s)}}{|x|^{s}} d x=0 .
$$

Thus, we have

$$
\begin{aligned}
o(1)\left\|u_{n}\right\| & =\left\|u_{n}\right\|^{p}-\int_{\Omega} \frac{\left|u_{n}\right|^{p^{*}(s)}}{|x|^{s}} d x-\lambda \int_{\Omega} f\left(x, u_{n}\right) u_{n} d x \\
& =\left\|u_{n}-u\right\|^{p}+\|u\|^{p}-\int_{\Omega} \frac{|u|^{p^{*}(s)}}{|x|^{s}} d x-\lambda \int_{\Omega} f(x, u) u d x \\
& =\left\|u_{n}-u\right\|^{p}+o(1)\|u\|,
\end{aligned}
$$

since $I^{\prime}(u)=0$. Thus we prove that $\left\{u_{n}\right\}$ strongly converges to $u$ in $H_{0}^{1, p}(\Omega)$.

\section{Existence of a sequence of arbitrarily small solutions}

In this section, we prove the existence of infinitely many solutions of (1.1) which tend to zero. Let $X$ be a Banach space and denote

$\Sigma:=\{A \subset X \backslash\{0\}: A$ is closed in $X$ and symmetric with respect to the orgin $\}$.

For $A \in \Sigma$, we define genus $\gamma(A)$ as

$$
\gamma(A):=\inf \left\{m \in N: \exists \varphi \in C\left(A, R^{m} \backslash\{0\}\right),-\varphi(x)=\varphi(-x)\right\} .
$$

If there is no mapping $\varphi$ as above for any $m \in N$, then $\gamma(A)=+\infty$. Let $\Sigma_{k}$ denote the family of closed symmetric subsets $A$ of $X$ such that $0 \notin A$ and $\gamma(A) \geq k$. We list some properties of the genus (see [16]).

Proposition 3.1. Let $A$ and $B$ be closed symmetric subsets of $X$ which do not contain the origin. Then the following hold.

(1) If there exists an odd continuous mapping from $A$ to $B$, then $\gamma(A) \leq \gamma(B)$;

(2) If there is an odd homeomorphism from $A$ to $B$, then $\gamma(A)=\gamma(B)$;

(3) If $\gamma(B)<\infty$, then $\gamma \overline{(A \backslash B)} \geq \gamma(A)-\gamma(B)$;

(4) Then $n$-dimensional sphere $S^{n}$ has a genus of $n+1$ by the Borsuk-Ulam Theorem;

(5) If $A$ is compact, then $\gamma(A)<+\infty$ and there exists $\delta>0$ such that $U_{\delta}(A) \in$ $\Sigma$ and $\gamma\left(U_{\delta}(A)\right)=\gamma(A)$, where $U_{\delta}(A)=\{x \in X:\|x-A\| \leq \delta\}$.

The following version of the symmetric mountain-pass lemma is due to Kajikiya [16].

Lemma 3.2. Let $E$ be an infinite-dimensional space and $I \in C^{1}(E, R)$ and suppose the following conditions hold. 
$\left(\mathrm{C}_{1}\right) I(u)$ is even, bounded from below, $I(0)=0$ and $I(u)$ satisfies the local Palais-Smale condition, i.e., for some $\bar{c}>0$, in the case when every sequence $\left\{u_{k}\right\}$ in $E$ satisfying $\lim _{k \rightarrow \infty} I\left(u_{k}\right)=c<\bar{c}$ and $\lim _{k \rightarrow \infty}\left\|I^{\prime}\left(u_{k}\right)\right\|_{E^{*}}=0$ has a convergent subsequence;

$\left(\mathrm{C}_{2}\right)$ For each $k \in N$, there exists an $A_{k} \in \Sigma_{k}$ such that $\sup _{u \in A_{k}} I(u)<0$.

Then either $\left(R_{1}\right)$ or $\left(R_{2}\right)$ below holds.

$\left(\mathrm{R}_{1}\right)$ There exists a sequence $\left\{u_{k}\right\}$ such that $I^{\prime}\left(u_{k}\right)=0, I\left(u_{k}\right)<0$ and $\left\{u_{k}\right\}$ converges to zero.

$\left(\mathrm{R}_{2}\right)$ There exist two sequences $\left\{u_{k}\right\}$ and $\left\{v_{k}\right\}$ such that $I^{\prime}\left(u_{k}\right)=0, I\left(u_{k}\right)<0$, $u_{k} \neq 0, \lim _{k \rightarrow \infty} u_{k}=0, I^{\prime}\left(v_{k}\right)=0, I\left(v_{k}\right)<0, \lim _{k \rightarrow \infty} v_{k}=0$, and $\left\{v_{k}\right\}$ converges to a non-zero limit.

Remark 3.3. In [16], the functional $I(u)$ is required to satisfy the Palais-Smale condition in global. However, if $I(u)$ satisfies the local Palais-Smale condition with the critical value levels $c \leq 0$, the results of Kajikiya's, i.e., [16, Theorem 1] remain true.

Remark 3.4. From Lemma 3.2 we have a sequence $\left\{u_{k}\right\}$ of critical points such that $I\left(u_{k}\right) \leq 0, u_{k} \neq 0$ and $\lim _{k \rightarrow \infty} u_{k}=0$.

In order to get infinitely many solutions we need some lemmas. Under the assumptions of Theorem 1.1, we take $\varepsilon=\frac{1}{\lambda_{1}}$ (where $\lambda_{1}$ is given in Sect. 1), then by the definition of $A_{s},(2.2)$ and Lemma 2.1 , for $\lambda \in\left(0, \frac{1}{\lambda_{1}}\right)$ we have

$$
\begin{aligned}
I(u)= & \frac{1}{p} \int_{\Omega}\left(|\nabla u|^{p}-\mu \frac{|u|^{p}}{|x|^{p}}\right) d x-\frac{1}{p^{*}(s)} \int_{\Omega} \frac{|u|^{p^{*}(s)}}{|x|^{s}} d x-\lambda \int_{\Omega} F(x, u) d x \\
\geq & \frac{1}{p} \int_{\Omega}\left(|\nabla u|^{p}-\mu \frac{|u|^{p}}{|x|^{p}}\right) d x-\frac{1+\lambda \varepsilon p^{*}(s)}{p^{*}(s)} \int_{\Omega} \frac{|u|^{p^{*}(s)}}{|x|^{s}} d x-\lambda b(\varepsilon)|\Omega| \\
\geq & \frac{1}{p} \int_{\Omega}\left(|\nabla u|^{p}-\mu \frac{|u|^{p}}{|x|^{p}}\right) d x \\
& -\frac{1+p^{*}(s)}{p^{*}(s)} A_{s}^{-p^{*}(s) / p}\left(\int_{\Omega}\left(|\nabla u|^{p}-\mu \frac{|u|^{p}}{|x|^{p}}\right) d x\right)^{\frac{p^{*}(s)}{p}}-\lambda b\left(\frac{1}{\lambda_{1}}\right) \\
= & A \int_{\Omega}\left(|\nabla u|^{p}-\mu \frac{|u|^{p}}{|x|^{p}}\right) d x-B\left(\int_{\Omega}\left(|\nabla u|^{p}-\mu \frac{|u|^{p}}{|x|^{p}}\right) d x\right)^{\frac{p^{*}(s)}{p}}-\lambda C,
\end{aligned}
$$

where

$$
A=\frac{1}{p}, \quad B=\frac{1+p^{*}(s)}{p^{*}(s)} A_{s}^{-p^{*}(s) / p}, \quad C=b\left(\frac{1}{\lambda_{1}}\right) .
$$

Let $Q(t)=A t^{p}-B t^{p^{*}(s)}-\lambda C$. Then

$$
I(u) \geq Q(\|u\|) .
$$

Furthermore, there exists

$$
\lambda_{*}=\min \left\{\lambda_{1}, \frac{A(p-s)}{C(N-s)}\left(\frac{p A}{p^{*}(s) B}\right)^{p /\left(p^{*}(s)-p\right)}\right\}>0
$$


such that for $\lambda \in\left(0, \lambda_{*}\right), Q(t)$ attains its positive maximum, that is, there exists

$$
R_{1}=\left(\frac{p A}{p^{*}(s) B}\right)^{1 /\left(p^{*}(s)-p\right)}
$$

such that

$$
e_{1}=Q\left(R_{1}\right)=\max _{t \geq 0} Q(t)>0 .
$$

Therefore, for $e_{0} \in\left(0, e_{1}\right)$, we may find $R_{0}<R_{1}$ such that $Q\left(R_{0}\right)=e_{0}$. Now we define

$$
\chi(t)= \begin{cases}1, & 0 \leq t \leq R_{0}, \\ \frac{A t^{p}-\lambda C-e_{1}}{B t^{* *}(s)}, & t \geq R_{1}, \\ C^{\infty}, \quad \chi(t) \in[0,1], & R_{0} \leq t \leq R_{1} .\end{cases}
$$

Then it is easy to see $\chi(t) \in[0,1]$ and $\chi(t)$ is $C^{\infty}$. Let $\varphi(u)=\chi(\|u\|)$ and consider the perturbation of $I(u)$ :

$G(u)=\frac{1}{p} \int_{\Omega}\left(|\nabla u|^{p}-\mu \frac{|u|^{p}}{|x|^{p}}\right) d x-\frac{\varphi(u)}{p^{*}(s)} \int_{\Omega} \frac{|u|^{p^{*}(s)}}{|x|^{s}} d x-\lambda \varphi(u) \int_{\Omega} F(x, u) d x$.

Then

$$
\begin{aligned}
G(u) & \geq A \int_{\Omega}\left(|\nabla u|^{p}-\mu \frac{|u|^{p}}{|x|^{p}}\right) d x-B \varphi(u)\left(\int_{\Omega}\left(|\nabla u|^{p}-\mu \frac{|u|^{p}}{|x|^{p}}\right) d x\right)^{\frac{p^{*}(s)}{p}}-\lambda C \\
& =\bar{Q}(\|u\|),
\end{aligned}
$$

where $\bar{Q}(t)=A t^{p}-B \chi(t) t^{p^{*}(s)}-\lambda C$ and

$$
\bar{Q}(t)=\left\{\begin{array}{l}
Q(t), 0 \leq t \leq R_{0} \\
e_{1}, \quad t \geq R_{1}
\end{array}\right.
$$

From the above arguments, we have the following:

Lemma 3.5. Let $G(u)$ is defined as in (3.1). Then

(i) $G \in C^{1}\left(H_{0}^{1, p}(\Omega), R\right)$ and $G$ is even and bounded from below;

(ii) If $G(u)<e_{0}$, then $\bar{Q}(\|u\|)<e_{0}$, consequently, $\|u\|<R_{0}$ and $I(u)=G(u)$;

(iii) There exists $\lambda^{*}$ such that, for $\lambda \in\left(0, \lambda^{*}\right), G$ satisfies a local $(P S)$ condition for

$$
c<e_{0} \in\left(0, \min \left\{e_{1}, \frac{p-s}{p(N-s)} A_{s}^{\frac{N-s}{p-s}}-\lambda c\left(\frac{p-s}{2 p \lambda(N-s)}\right)|\Omega|\right\}\right) .
$$

Proof. It is easy to see (i) and (ii). (iii) are consequences of (ii) and Lemma 2.2.

Lemma 3.6. Assume that $\left(H_{3}\right)$ of Theorem 1.1 holds. Then for any $k \in N$, there exists $\delta=\delta(k)>0$ such that $\gamma\left(\left\{u \in H_{0}^{1, p}(\Omega): G(u) \leq-\delta(k)\right\} \backslash\{0\}\right) \geq k$. 
Proof. Firstly, by $\left(H_{3}\right)$ of Theorem 1.1, for any fixed $u \in H_{0}^{1, p}(\Omega), u \neq 0$, we have

$$
F(x, \rho u) \geq M(\rho)(\rho u)^{p} \quad \text { with } \quad M(\rho) \rightarrow \infty \text { as } \rho \rightarrow 0 .
$$

Secondly, given any $k \in N$, let $E_{k}$ be a $k$-dimensional subspace of $H_{0}^{1, p}(\Omega)$. There then exist constant $\sigma_{k}$ such that

$$
\|u\| \leq \sigma_{k}|u|_{p}, \quad \forall u \in E_{k}
$$

Therefore for any $u \in E_{k}$ with $\|u\|=1$ and $\rho$ small enough, we have

$$
\begin{aligned}
G(\rho u) & =I(\rho u) \\
& \leq \frac{\rho^{p}}{p} \int_{\Omega}\left(|\nabla u|^{p}-\mu \frac{|u|^{p}}{|x|^{p}}\right) d x-\frac{\rho^{p^{*}(s)}}{p^{*}(s)} \int_{\Omega} \frac{|u|^{p^{*}(s)}}{|x|^{s}} d x-\lambda M(\rho) \int_{\Omega}|u|^{p} d x \\
& \leq\left(\frac{1}{p}-\frac{\lambda M(\rho)}{\sigma_{k}^{p}}\right) \rho^{p} \\
& =-\delta(k)<0
\end{aligned}
$$

since $\lim _{|\rho| \rightarrow 0} M(\rho)=+\infty$. That is,

$$
\left\{u \in E_{k}:\|u\|=\rho\right\} \subset\left\{u \in H_{0}^{1, p}(\Omega): G(u) \leq-\delta(k)\right\} \backslash\{0\} .
$$

This completes the proof.

Now we give the proof of Theorem 1.1 as following.

Proof of Theorem 1.1. Recall that

$$
\Sigma_{k}=\left\{A \in H_{0}^{1, p}(\Omega) \backslash\{0\}: A \text { is closed and } A=-A, \gamma(A) \geq k\right\}
$$

and define

$$
c_{k}=\inf _{A \in \Sigma_{k}} \sup _{u \in A} G(u) .
$$

By Lemmas 3.5 (i) and 3.6, we know that $-\infty<c_{k}<0$. Therefore, assumptions $\left(C_{1}\right)$ and $\left(C_{2}\right)$ of Lemma 3.2 are satisfied. This means that $G$ has a sequence of solutions $\left\{u_{n}\right\}$ converging to zero. Hence, Theorem 1.1 follows by Lemma 3.5 (ii).

\section{Acknowledgments}

The authors of this paper wish to thanks the referee for valuable suggestions regarding the original manuscript. The authors also would like to thank Professor H. Yin for his help and many valuable discussions. The Project was supported by NSFC(10871096), the project of Nanjing Normal University and Project of Graduate Education Innovation of Jiangsu Province(181200000214). 


\section{References}

[1] Brezis, H., Nirenberg, L.: Positive solutions of nonlinear elliptic equations involving critical exponents. Comm. Pure Appl. Math. 34, 437-477 (1983)

[2] He, X.M., Zou, W.M.: Infinitely many arbitrarily small solutions for sigular elliptic problems with critical Sobolev-Hardy exponents. Proc. Edinb. Math. Soc. 52, 97-108 (2009)

[3] Terracini, S.: On positive solutions to a class equations with a singular coefficient and critical exponent. Adv. Differ. Equ. 2, 241-264 (1996)

[4] Ferrero, A., Gazzola, F.: Existence of solutions for singular critical growth semilinear elliptic equations. J. Differ. Equ. 177, 494-522 (2001)

[5] Cao, D., Han, P.: Solutions for semilinear elliptic equations with critical exponents and Hardy potential. J. Differ. Equ. 205, 521-537 (2004)

[6] Cao, D., Han, P.: Solutions to critical elliptic equations with multi-singular inverse square potentials. J. Differ. Equ. 224, 332-372 (2006)

[7] Cao, D., He, X., Peng, S.: Positive solutions for some singular critical growth nonlinear elliptic equations. Nonlinear Anal. 60, 589-609 (2005)

[8] Cao, D., Peng, S.: A note on the sign-changing solutions to elliptic problems with critical Sobolev and Hardy terms. J. Differ. Equ. 193, 424-434 (2003)

[9] Ghoussoub, N., Yuan, C.: Multiple solutions for quasi-linear PDEs involving the critical Sobolev and Hardy exponents. Trans. Am. Math. Soc. 352, 5703-5743 (2000)

[10] Chabrowski, J.: On multiple solutions for the nonhomogeneous $p$-Laplacian with a critical Sobolev exponent. Differ. Integ. Equ. 8, 705-716 (1995)

[11] Silva, E.A., Xavier, M.S.: Multiplicity of solutions for quasilinear elliptic problems involving critical Sobolev exponents. Ann. Inst. H. Poincaré Anal. NonLinéaire 20, 341-358 (2003)

[12] Filippucci, R., Pucci, P., Robert, F.: On a $p$-Laplace equation with multiple critical nonlinearities. J. Math. Pures Appl. 91, 156-177 (2009)

[13] Musina, R.: Existence and multiplicity results for a weighted $p$-Laplace equation involving Hardy potentials and critical nonlinearities. Rend. Lincei Mat. Appl. 20, 127-143 (2009)

[14] Kang, D.S.: On the quasilinear elliptic problems with critical Sobolev-Hardy exponents and Hardy terms. Nonlinear Anal. 68, 1973-1985 (2008)

[15] Chen, J., Li, S.: On multiple solutions of a singular quasi-linear equation on unbounded domain. J. Math. Anal. Appl. 275, 733-746 (2002)

[16] Kajikiya, R.: A critical-point theorem related to the symmetric mountain-pass lemma and its applications to elliptic equations. J. Funct. Anal. 225, 352$370(2005)$ 
[17] Caffarelli, L., Kohn, R., Nirenberg, L.: First order interpolation inequality with weights. Compos. Math. 53, 259-275 (1984)

[18] Garcia Azorero, J., Peral, I.: Hardy inequalities and some critical elliptic and parabolic problems. J. Differ. Equ. 144, 441-476 (1998)

[19] Rabinowitz, P.H.: Minimax methods in critical-point theory with applications to differential equations. In: CBME Regional Conference Series in Mathematics, vol. 65. American Mathematical Society, Providence (1986)

[20] Li, S., Zou, W.: Remarks on a class of elliptic problems with critical exponents. Nonlinear Anal. 32, 769-774 (1998)

[21] Lions, P.L.: The concentration-compactness principle in the caculus of variation: the limit case, I. Rev. Mat. Iberoamericana 1, 45-120 (1985)

[22] Lions, P.L.: The concentration-compactness principle in the caculus of variation: the limit case, II. Rev. Mat. Iberoamericana 1, 145-201 (1985)

[23] Brezis, H., Lieb, E.: A relation between pointwise convergence of functions and convergence of functionals. Proc. Am. Math. Soc. 88, 486-490 (1983)

[24] Smets, D.: A concentration-compactness principle lemma with applications to singular eigenvalue problems. J. Funct. Anal. 167, 463-480 (1999)

S. Liang $(\bowtie)$, J. Zhang

School of Mathematical Sciences,

Institute of Mathematics,

Nanjing Normal University,

210097 Jiangsu,

People's Republic of China

e-mail: liangsihua@163.com

J. Zhang

e-mail: jihuiz@jlonline.com

S. Liang

College of Mathematics,

Changchun Normal University,

130032 Changchun, Jilin,

People's Republic of China

Received: 16 March 2009.

Accepted: 02 September 2009. 